MITSUBISHI ELECTRIC RESEARCH LABORATORIES

http://www.merl.com

\title{
Direct-Touch vs. Mouse Input for Tabletop Displays
}

\author{
Clifton Forlines, Daniel Wigdor, Chia Shen, Ravin Balakrishnan
}

TR2007-053 April 2007

\begin{abstract}
We investigate the differences - in terms of both quantitative performance and subjective preference - between direct-touch and mouse input for unimanual and bimanual tasks on tabletop displays. The results of two experiments show that for bimanual tasks performed on tabletops, users benefit from direct-touch input. However, our results also indicate that mouse input may be more appropriate for a single user working on tabletop tasks requiring only single-point interaction.
\end{abstract}

Conference on Human Factors in Computer Systems (CHI)

This work may not be copied or reproduced in whole or in part for any commercial purpose. Permission to copy in whole or in part without payment of fee is granted for nonprofit educational and research purposes provided that all such whole or partial copies include the following: a notice that such copying is by permission of Mitsubishi Electric Research Laboratories, Inc.; an acknowledgment of the authors and individual contributions to the work; and all applicable portions of the copyright notice. Copying, reproduction, or republishing for any other purpose shall require a license with payment of fee to Mitsubishi Electric Research Laboratories, Inc. All rights reserved.

Copyright (C) Mitsubishi Electric Research Laboratories, Inc., 2007

201 Broadway, Cambridge, Massachusetts 02139 



\section{Direct-Touch vs. Mouse Input for Tabletop Displays}

\author{
Clifton Forlines $^{1,2} \quad$ Daniel Wigdor ${ }^{1,2}$ \\ ${ }^{1}$ Mitsubishi Electric Research Labs \\ Cambridge, MA, USA \\ www.merl.com \\ forlines | shen@merl.com
}

\begin{abstract}
We investigate the differences - in terms of both quantitative performance and subjective preference between direct-touch and mouse input for unimanual and bimanual tasks on tabletop displays. The results of two experiments show that for bimanual tasks performed on tabletops, users benefit from direct-touch input. However, our results also indicate that mouse input may be more appropriate for a single user working on tabletop tasks requiring only single-point interaction.
\end{abstract}

\section{Author Keywords}

Tabletop computing, direct-touch interfaces, bimanual input, multiple mice.

\section{ACM Classification Keywords}

H.5.2 Information interfaces and presentation (e.g., HCI): User Interfaces Input devices and strategies (e.g., mouse, touchscreen)

\section{INTRODUCTION}

Direct-touch interactive tabletop displays have been the focus of numerous research projects $[11,34,36,37,38,41]$ and appear to provide several benefits over traditional desktop displays. One of the most often argued benefits is the notion that interacting with an application through directly touching graphical elements is a more "natural" or "compelling" approach than working indirectly with a mouse or other pointing device. While "naturalness" is difficult to measure, generally implicit in this argument is that this affordance might also result in improved efficiency and accuracy. In traditional desktop display settings, however, there is some evidence [31] that indirect mouse input may equal or outperform direct-touch input when the task requires just a single point of contact. Furthermore, it is difficult to accurately point at objects that are smaller than one's finger, although several researchers $[1,2,6,11,39$, 40] have developed clever techniques to alleviate this issue. These studies comparing indirect mouse input with direct-

Permission to make digital or hard copies of all or part of this work for personal or classroom use is granted without fee provided that copies are not made or distributed for profit or commercial advantage and that copies bear this notice and the full citation on the first page. To copy otherwise, or republish, to post on servers or to redistribute to lists, requires prior specific permission and/or a fee.

CHI 2007, April 28-May 3, 2007, San Jose, California, USA

Copyright 2007 ACM 978-1-59593-593-9/07/0004...\$5.00.

\author{
Chia Shen $^{1}$ Ravin Balakrishnan ${ }^{2}$ \\ ${ }^{2}$ Department of Computer Science \\ University of Toronto \\ www.dgp.toronto.edu \\ dwigdor | ravin@dgp.toronto.edu
}

touch input, and the resulting design solutions, have generally been conducted with vertical desktop displays. The applicability of these results and designs to larger horizontal tabletop displays remains to be investigated.

Another open question is the performance implications of bimanual and gestural interaction using direct vs. indirect input devices. Research systems [41] have demonstrated whole-hand bimanual gestural interaction on tabletop displays that appear to take advantage of the more "natural" and higher bandwith input provided by direct-touch. However, if one's intention is to support just two points of interaction as opposed to multiple points, direct-touch sensing is not necessarily required - at least from a technological standpoint - since bimanual input is easily and inexpensively supported through the addition of an extra mouse to a typical desktop computer. From a human physiological standpoint, proprioception, one's inherent ability to keep track of the location of one's body parts kinesthetically might be expected to result in significant advantages for direct-touch bimanual input; however, it is unclear whether occlusion and the reaching over large distances on a tabletop will counteract this benefit. Furthermore, it has been shown [3] that as long as appropriate visual feedback is present, indirect input devices perform well despite perturbations to the user's kinesthetic reference frames. However, when using two mice, questions remain as to whether or not a user can successfully track two graphical pointers [4] on a large tabletop display, and if a user can successfully manage multiple mice with the dynamic control-display mappings [32] commonly used in today's single mouse systems.

Given these open questions, a systematic investigation is clearly needed to help system designers choose the most appropriate input mechanism when designing interfaces for tabletop displays. In this paper, we present two experiments designed to investigate the differences - in terms of both quantitative performance and subjective preference between direct-touch and mouse input for unimanual and bimanual tasks on tabletop displays. Our results not only indicate that users would benefit from a direct-touch tabletop when performing bimanual tasks, but also raise questions as to the appropriateness of a direct-touch tabletop interface for a single user working on tasks requiring only single-point interaction 


\section{RELATED WORK}

Card, English, and Burr [9] found that indirect mouse input compared very favorably to direct stylus input in their seminal work on the quantitative comparison of pointing devices. While there are important differences between stylus and touch input, one would expect that these results might generalize to single-finger pointing. Sears and Shneiderman [39] compared mouse input to touch screen input in a unimanual task. Their experiment used a $27.6 \times 19.5 \mathrm{~cm}$ vertically oriented display with a resolution of $640 \times 480$ pixels, with resulting pixel size of $0.4 \mathrm{~mm}^{2}$. They found that for targets 16 pixels or more in width, touchscreen selection was faster than mouse selection. Further, for targets 32 pixels in width, touch screen selection resulted in about $66 \%$ fewer errors. Yet, even with the apparent superior performance of direct-touch input, participants still preferred mouse input. Several important features may limit the applicability of these findings to tabletop displays. The study used a mouse with a controldisplay gain of approximately $1: 1$, which is rare nowadays and would be inappropriate for a table-sized display. Additionally, a dynamic control-display gain based on mouse velocity has become quite popular in desktop GUIs. Indeed, WindowsXP ships with this ballistic mouse pointing enabled by default [32]. Perhaps most importantly, Sears and Shneiderman used a vertically oriented touch screen, which may have important differences from a horizontal table in terms of reach and target visibility.

Meyer et al. [31] compared user performance with two absolute (direct-touch screen, indirect stylus) and three relative devices (mouse, trackball, mousepen) on a desktop display. They found that the mouse resulted in the best performance while the touch screen resulted in the worst performance. In fact, they found all absolute input devices to be slower than the relative devices and concluded that "relative mapping is superior to absolute mapping." This experiment used a small vertical display, which has important differences from a large touch-table. These results are in contrast to that of Accot and Zhai [1] who found that for steering tasks users were about twice as fast with an 8 "x6" tablet in absolute mode than with a smaller indirect touchpad in relative mode.

Parker et al. [36] presented the "Tractor-Beam" input device, a stylus tracked in 3D and used like a laser pointer. When held against the table surface, the device acts as an absolute stylus. Through lifting and returning the pen tip to the table, the user switches between direct and indirect input; however, accurate selection of distant targets with a laser pointer is error prone making the usability of this device questionable for some tasks on large displays.

Albinsson and Zhai [2] compared a collection of input techniques designed to improve the accuracy of bare hand interaction with a touch screen. They found different rankings of the techniques along performance and preferential lines depending on task and target size. They conclude that system designers should provide the user with a variety of selection tools so that the user can choose the most appropriate tool for the task at hand.

Recent works by Benko et al. [6] and Esenther et al. [11] have done just this. These authors investigated the use of additional fingers to mode the mapping between touches and control point on tabletop displays. In this manner, a user is able to switch to a slower, more accurate mapping when detailed control is needed, and can default back to direct single-finger input when working with larger targets.

There has been significant research in the area of bimanual interaction, in terms of theory $[14,15]$, empirical studies $[8$, $19,20,21,22,25,30]$, and interaction design $[6,17,18,33$, 42]. Amongst the earliest work in the HCI field is the study of Buxton and Myers [8], which clearly articulated the benefits of bimanual input on graphical user interface tasks. This body of research has typically investigated bimanual interaction with desktop displays or in virtual reality environments, but not in tabletop settings.

Of particular relevance to our current work is a study by Balakrishnan and Hinckley [3] that investigated the value of proprioception in asymmetric bimanual tasks. They found that users benefit from working in a single absolute reference frame when completing bimanual tasks when visual feedback is absent, but that the benefit diminishes when visual feedback is provided. While their experiment did include a two-mouse condition, it was not intended to provide a comparison between two-mouse and two-pen input per se. The mice used in this experiment had a fixed $\mathrm{C}: \mathrm{D}$ gain ratio of $1: 1$, and participants often had to "clutch" the mice in order to complete a task.

Latulipe et al. [23, 24] have published a series of investigations into symmetric bimanual input performed with two mice on a desktop display. They found that for many tasks, symmetric bimanual input outperforms not only single mouse, but also asymmetric bimanual input in terms of performance and preference, and advocate the addition of a second mouse to desktop computer systems. Perhaps most similar to our work, Barnert [5] describes an experiment in which participants performed better when using a pair of mice than when using two hands directly on a table while completing an asymmetric bimanual task. Because the task used in this study required pixel-accurate positioning, the author suggests that the superior performance of the mice may be due to the relatively large size and low accuracy of one's fingertips.

In summary, our survey reveals considerable research in the areas of tabletop interaction, bimanual input, and direct vs. indirect input. However, there has yet to be a systematic investigation as to the relative merits of direct touch vs. indirect mouse input for tabletop displays for unimanual and symmetric bimanual tasks. Given the exciting recent and ongoing activity in the area of tabletop interaction, we believe it is crucial to obtain a better understanding of the pros and cons of using these different input mechanisms for interacting with this relatively new display form factor. 


\section{EXPERIMENT 1 - UNIMANUAL INPUT}

In our first experiment, our goal was to compare singlefinger, direct-touch input with single mouse, indirect input for selection and dragging operations on a tabletop display. Based on previous comparisons between direct input and mouse input for smaller displays [9, 39], we hypothesized that we would observe no performance difference in terms of selection speed. Occlusion becomes a large problem when selecting small targets with one's finger; therefore, we also hypothesized that direct-touch input would result in more selection errors than mouse input.

Twelve people ( 2 female, 10 male) were recruited from the local community via mailing lists. Participants ranged in age from 22 to 47 years. All participants were right-handed, with the exception of one who was left-handed but normally used his mouse with his right hand. Participants were paid $\$ 10$ in compensation for their time.

\section{Apparatus}

Participants sat in front of a horizontal, top-projected, 107 centimeter diagonal MERL DiamondTouch touch sensitive table (Figure 1). The resolution of the projected display was $1024 \times 768$ pixels with each pixel approximately $1.2 \mathrm{~mm}$ square. The DiamondTouch table is capable of detecting finger touches at a resolution of $0.4 \mathrm{~mm}$. The physical table included an inactive border region that was not projected on. This region included a mouse-pad during the mouse input trials. We used an 800 dpi optical Microsoft mouse with ballistic pointing enabled and set to a comfortable level such that mouse clutching was never needed.



Figure 1. In Expt. 1, participants used a finger or a mouse to select and drag targets. In Expt. 2, they used either two mice or two fingers to perform a symmetric bimanual docking task.

\section{Task}

We used a traditional 2D target selection and docking task. By requiring both selection and dragging actions, our task is a representative abstraction of tasks commonly performed in graphical interfaces. While a Fitts' [12] style selection task alone is commonly used in the literature to comparatively evaluate input techniques, it does not provide insights into how the techniques perform for the arguably more demanding dragging actions that form a significant subset of graphical interaction. In particular, when dragging with direct-touch input, the user must keep their finger in contact with the display surface for extended periods of time - a requirement that is not present in selection tasks. This additional task complexity might reveal differences between input techniques that might not be apparent in the simpler selection task.

Before each trial, the participant placed their finger or mouse pointer within a home location at the near edge of the tabletop (Figure 2). To prevent participants from anticipating the start of a trial, the test application delayed the presentation of the target and dock by a random amount of time after the selection of the home location (between $0.5 \mathrm{~s}$ and $2 \mathrm{~s}$ ). If the participant lifted their finger from the display or moved their mouse pointer out of the home location, the trial would not start and an error sound was played until the participant returned to the home location.

At the beginning of a trial, a green target and a grey dock would appear on the display (Figure 2). The distance between the home location and target was equal to the distance between the target and dock. The participant was asked to "as quickly and as accurately as possible" select the target and drag it into the dock. Docking occurred automatically once the center of the target had been dragged to within 5 pixels of the center of the dock and did not require the lifting of a finger or the release of the mouse button. We have found that this variation on the docking task effectively removes any between-participant differences in how accurately individuals feel they have to align a target with a dock. After a successful docking, the dock and target would disappear and the home location marker would again appear near the user.



Figure 2. Task details. (left) Round home location, the target on the right, and the dock on the left. (right) After selecting the target, participants dragged it to the dock.

Whenever the participant missed the target, an error sound was played and a selection error recorded. A docking error occurred whenever the participant released the target before it was successfully docked. To prevent participants from racing through the experimental trials without regard for accuracy, they would not move onto the next trial until a successful selection and docking had been performed, even in the case of multiple selection and docking errors. This design results in participants having to make a reasonable optimization between speed and accuracy. 


\section{Design}

We used a repeated-measures design with the withinparticipant independent variables input device (mouse and touch-table), target width (16, 32, 48, and 64 pixels), and target distance (300, 400, 500, and 600 pixels). These target widths and distances correspond to a range of Fitts' Law [12] index of difficulties from 2.5 to 5.3. Each participant performed 2 blocks of trials for each input device. Within each block, 4 selection and docking actions were made for each of the 16 target width and target distance combinations. The width and distance presentation was randomized. The order of presentation of the two input devices was counterbalanced among participants, and all participants were presented with the same set of target and dock locations. Participants could take breaks after any trial, and experimental sessions lasted about 45 minutes. In summary, the design was:

\section{2 participants $\mathrm{x}$}

2 input devices (mouse, touch-table) $\mathrm{x}$

2 blocks of trials $\mathrm{x}$

4 target widths $(16,32,48,64$ pixels $) x$

4 target distances $(300,400,500,600$ pixels $) \mathrm{x}$

4 repetitions

$=3072$ selections in total.

The dependent variables measured were selection time, docking time, overall trial time (selection + docking), selection errors, and docking errors.

\section{Results}

\section{Selection Time Analysis}

Selection-time was measured as the time between the presentation of a target and the successful selection of that target. If a participant initially missed a target, they had to continue to try to select the target in order to move through the experiment. An ANOVA of the collected data shows a strong main effect of input device on selection time $\left(\mathrm{F}_{1,11}=\right.$ $66.65, \mathrm{p}<0.001$ ), with mean selection times of $1.01 \mathrm{~s}$ and $1.19 \mathrm{~s}$ for touch-table and mouse input respectively.

Independent analysis of width and distance in a pointing task should be considered judiciously [13], since width and distance are not independent factors - which is an assumption of an ANOVA. However, an analysis of width and distance often provides insight. As one would expect in a pointing task, both width and distance had strong main effects on selection-time $\left(\mathrm{F}_{3,33}=350.93, \mathrm{p}<0.001\right.$ and $\mathrm{F}_{3,33}$ $=67.69, \mathrm{p}<0.001$ for width and distance respectively), with smaller targets and more distant targets taking longer to select than larger and closer ones. Interestingly, while there was no significant interaction between input device and distance $\left(\mathrm{F}_{3,33}=0.71, \mathrm{p}=0.55\right)$, there was a strong interaction between input device and width $\left(\mathrm{F}_{3,33}=21.29\right.$, $\mathrm{p}$ $<0.001)$. While smaller targets always had longer selection times, touch-table selection times increased dramatically as targets shrank from 32 to 16 pixels. Figure 3 shows the mean selection times for both input devices for each width.



Figure 3. Selection times for each width and input device.

Fitts-law Analysis of the Selection Portion of the Task

The performance of a pointing technique can be modeled with the Shannon formulation of Fitts' law $[12,26]$. The index of difficulty $(I D)$ of a pointing task is a function of target distance $(D)$ and target width $(W)$, and movement time $(M T)$ can be predicted:

$$
M T=a+b I D, \text { where } I D=\log _{2}\left(\frac{D}{W}+1\right)
$$

where $a$ and $b$ are specific to a certain technique and are found using linear regression. The reciprocal of $b$ has been termed the index of performance $(I P)$ and traditionally used in the literature $[12,26]$ as a measure of the technique's throughput, with a higher $I P$ indicating a more efficient technique. However, Zhai [43] has recently argued that $I P$ is lacking in several aspects, and incorrectly reduces a multi variable measurement into a single measure. Thus, although we compute and present $I P$ measures, this should be taken within the context of Zhai's recent clarifications.

Although Fitts' law was originally formulated for 1D tasks, it has since been generalized to $2 \mathrm{D}$ and $3 \mathrm{D}$ tasks with various techniques for computing the "width" of these higher degree-of-freedom targets [1, 16, 27]. Because our targets had an equal width and height, we could simply do our analysis using width directly. Table 1 summarizes the Fitts' law parameters for both input devices. The data used to construct these models does not include trials marked as errors. The high $r^{2}$ values indicate a close fit with the linear model. It is interesting to note that the IP calculated for our mouse data is very similar to that of previous work performed on desktop displays [29].

\begin{tabular}{|c|c|c|c|}
\hline Input-device & Model & IP & $\boldsymbol{r}^{2}$ \\
\hline Mouse & $0.28+0.23 *$ ID & 4.35 & 0.97 \\
\hline Touch-Table & $0.46+0.12 *$ ID & 8.05 & 0.93 \\
\hline
\end{tabular}

Table 1. Fitts model, Index of Performance, and linear fit for each input-device.

Selection Error Analysis

A selection error occurred whenever the participant missed the target on their first attempt. An ANOVA of the recorded data shows a significant effect for input device on selection error $\left(F_{1,11}=13.46, p=0.004\right)$, with participants being about twice as likely to commit a selection error using the touch-table $(8.5 \%)$ than using the mouse $(4.1 \%)$. Target 
width had a significant effect on selection error $\left(\mathrm{F}_{3,33}=\right.$ 103.56, $\mathrm{p}<0.001)$, as did distance $\left(\mathrm{F}_{3,33}=13.68, \mathrm{p}<\right.$ $0.001)$. Input device significantly interacted with both width $\left(\mathrm{F}_{3,33}=14.43, \mathrm{p}<0.001\right)$ and distance $\left(\mathrm{F}_{3,33}=16.28, \mathrm{p}<\right.$ $0.001)$, and there was a significant interaction among input device, width, and distance $\left(\mathrm{F}_{9,99}=3.83, \mathrm{p}<0.001\right)$.

\section{Docking Time Analysis}

An ANOVA shows a strong main effect for input device on docking time $\left(\mathrm{F}_{1,11}=17.36, \mathrm{p}=0.002\right)$, with mean docking times of $1.09 \mathrm{~s}$ and $0.92 \mathrm{~s}$ for touch-table and mouse input respectively. Both target width and distance had significant main effects on docking time $\left(\mathrm{F}_{3,33}=13.75, \mathrm{p}<0.001\right.$ and $\mathrm{F}_{3,33}=36.75, \mathrm{p}<0.001$ for width and distance respectively). Note that the effect for width is somewhat surprising since the threshold for docking of 5 pixels was identical across all target/dock widths. This might be explained by the significant interaction between width and input device $\left(\mathrm{F}_{3,33}\right.$ $=9.43, \mathrm{p}<0.001)$. Pairwise post-hoc means comparisons show significant difference between the smallest and every other width, and Figure 4 shows that this difference is entirely attributable to touch-table input. There was also a distance and input device interaction $\left(\mathrm{F}_{3,33}=3.81, \mathrm{p}=0.02\right)$, with pairwise means comparisons showing a significant difference between all possible pairs of distances. Figure 5 shows that the performance difference between touch-table and mouse docking grows with target distance.



Figure 4. Docking times for each width and input device.



Figure 5. Docking times for each distance and input device.

\section{Docking Error Analysis}

Overall, there was little difference between devices in terms of docking error $\left(\mathrm{F}_{1,11}=0.02, \mathrm{p}=0.90\right)$ with mean error rates of $2.7 \%$ and $2.6 \%$ for touch-table and mouse input.

\section{Trial Time Analysis}

Overall, the mean trial times for both input devices were almost identical, with mean trial times of $2.12 \mathrm{~s}$ and $2.13 \mathrm{~s}$ for touch-table and mouse input respectively. The superior performance of touch-table input in terms of selection time was equally countered by the superior performance of mouse input in terms of docking time, as shown in Figure 6.



Figure 6. Mean unimanual trial times for each input device, broken down by selection time and docking time.

\section{Preferential Analysis}

We asked participants which input device they preferred for this task. While a Wilcoxon signed rank test did not indicate that there was a significant difference in preference $(Z=-1.7, p=0.08), 9$ of 12 participants chose the mouse as their preferred input device for this unimanual task.

\section{Experiment 1 Discussion}

In terms of selection time, our results reinforce Sears and Shneiderman's [39] in that direct-touch and mouse input had very similar performance, with touch input slightly outperforming mouse input. Figure 7 shows Buxton's threestate model for graphical input [7]. MacKenzie et al. [28] showed that State 2 dragging movement is slower than State 1 tracking movement for a variety of input devices. While our participants' docking times were faster than their selection times, our docking task ended once the target was "close enough" and is thus not directly comparable to MacKenzie's State 2 targeting task that required the participant to release the mouse button once the pointer was within the target. In our experiment, State 2 mouse movement was faster than State 2 touch-table movement, and this difference increased with an increase in docking distance (Figure 5), indicating that dragging one's finger across a table is an inefficient means of State 2 input when compared to dragging a target with a mouse.

The significant effect of target width on docking time, despite the identical 5 pixel docking threshold across all target widths, is most likely explained by the interaction between input device and target width. Touch-table docking was particularly problematic for the smallest target width (Figure 4), during which the participant's finger completely obscured the dock projected on the table.



Figure 7: Three-state model for graphical input [7]. 
While selection was faster for touch-table input, error rates were much higher. In the experimental task, selection errors had little consequence beyond the lengthening of a trial due to having to select that target again. Even with these multiple attempts at selection included in mean selection times, touch-table input still came out ahead. However, were selection errors to incur a greater penalty (such as accidentally closing a window instead of minimizing it), we should expect to see longer task times for touch-table input as users worked more carefully to avoid these costly errors.

In regards to selection error, the interaction between input device and target width, as well as the interaction between input device and target distance is likely explained by the three-way interaction among input device, target width, and target distance. Figure 8 shows the error rate for both input devices for each target distance for targets with a width of 16 pixels (the error rates for other width/distance/input combinations were small and very similar). The touch-table error rate for these small targets was zero when they were close to the user, and grew as they became more distant.



Figure 8. Selection error rates for each target distance and input device for targets with a width of 16 pixels.

We hypothesize that this is due to a combination of two factors. The first is the perspective distortion that occurs on very large displays when the distance between the graphical object and the user becomes large. Czerwinski et al. [10] proposed that large displays curve around the user to minimize this type of distortion, but this solution may be inappropriate for a horizontal display surrounded by a team of people each of whom has a different point of view.

The second factor comes from an understanding of the physical contact between a finger and touch sensitive surface and how this contact changes for differently distanced targets. Figure 9 shows a user touching two locations on a small vertical touch screen. In all locations, the interface between the finger-tip and screen is similar, with the index finger perpendicular to the display and the other fingers not in danger of committing accidental input. The contact patch of the fingertip maintains a relatively consistent shape, an important factor if the point of contact is taken as the centroid of the touch area as it results in a consistent point of interaction relative to the finger-tip for all touches on the display.

Figure 10 shows a user touching several locations on a large tabletop display. At locations close to the user, the interface between the finger and table is very similar to the interface between a finger and vertically oriented touchscreen; however, problems arise as distant targets are selected. The shape of the contact area between finger and table changes for different areas of the table (Figure 11), and at some distances, other fingers are in danger of providing accidental input to the system (Figure 10, bottom).

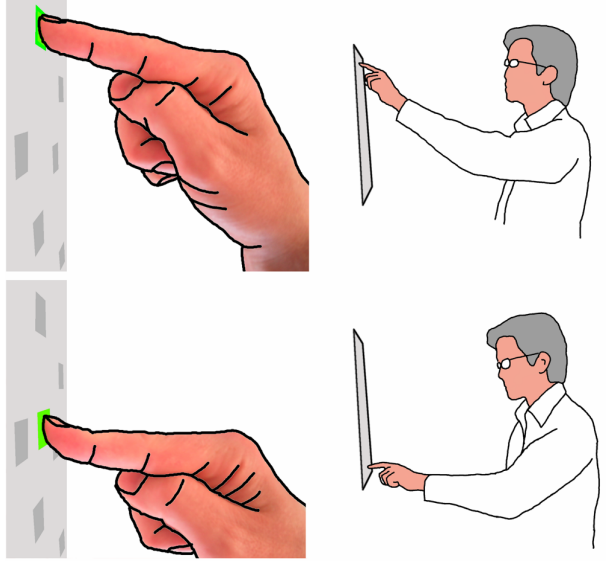

Figure 9. The contact between a finger and vertical touchscreen is similar for touches in any location.



Figure 10. The contact between a finger and the tabletop changes for touches in different locations on the table. Additionally, non-index fingers are in danger of providing accidental input at some distances (bottom).


Figure 11. The changing contact area results in a non-uniform mapping between fingertip and point of interaction.

These findings are important as they indicate the need for designers of graphical widgets for tabletop interaction to account for these differences in performance due to the distance of the activation zone from the user. These differences in the contact between finger and display at different locations on the tabletop may explain the higher error rates for touch input in our experiment than those found by Sears and Shneiderman [39]. The targets at a distance of 300 pixels resulted in a contact with the table that was most like the vertical display used in this previous work, and selection errors were very low at this distance. 


\section{EXPERIMENT 2 - BIMANUAL INPUT}

In our second experiment, we compared direct-touch and multiple mouse input for a symmetric bimanual task. The ease with which a second mouse can be added to a computer system makes it an appealing option for supporting bimanual input; however, it is unclear whether users can successfully manage two graphical pointers [4]. Given potential benefits of proprioception between hands in bimanual tasks [3], we hypothesized that direct-touch input would result in faster selection times than multiple mouse input. Again because of the problem with occlusion, we also hypothesized that direct-touch input would result in a greater number of selection errors in our bimanual task.

The same 12 individuals who participated in Experiment 1 took part in Experiment 2. The experiment lasted about 45 minutes, and participants were paid $\$ 10$. Participants sat in front of the same touch-table used in Experiment 1. For the multiple-mice conditions, two mouse pads were placed on inactive regions of the table. Both mice used the same ballistic pointer acceleration used in Experiment 1.

\section{Task}

We used a 2D bimanual target selection, resize and docking task. By requiring resizing of the target after acquisition, this task adds important interaction complexity and seamlessly builds upon the more elemental task used in Experiment 1. The symmetric nature of the bimanual interaction [4] arguably would also enable the user to take advantage of proprioception where available.

At the beginning of a trial, a green target and a grey dock of a different size would appear on the display (Figure 12). The participant was asked to "as quickly and as accurately as possible" select the target by selecting the handles on its opposite corners and resize and position it over the dock. These handles had a radius of 16 pixels. Docking occurred automatically once all sides of the target were within 5 pixels of the dock and did not require lifting one's fingers or the release of the mouse buttons. After a successful docking, the dock and target would disappear and the pair of markers indicating the home locations was again displayed near the participant. Participants were allowed to practice the task for as long as they wished before starting the experimental trials for each of the input devices.

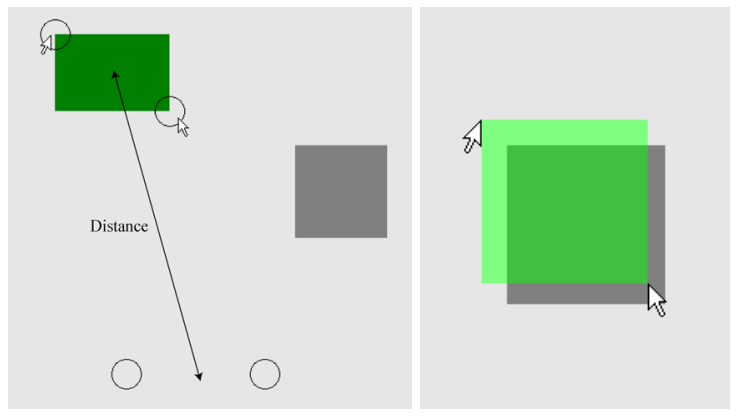

Figure 12. (left) Participants selected targets by selecting opposite corners. (right) Participants then dragged and resized the target so that it matched the dock.
A selection error occurred whenever the participant missed either of the corner handles of the target, and an error sound was played. A docking error occurred whenever the participant released either corner of the target before it was successfully docked. As in Experiment 1, to prevent participants from racing through the experimental trials, the testing application would not move onto the next trial until a successful selection and docking had been performed, even in the case of multiple selection and docking errors.

\section{Design}

We used a repeated-measures design with the withinparticipant independent variables input device (mouse and touch-table). Each participant performed 8 blocks of trials for each input device. Within each block, 16 select, resize and dock actions were made on targets placed at a distance between 100 and 600 pixels from the home location with an initial diagonal size between 75 and 400 pixels. The variables measured were selection time, docking time, trial time (selection + docking), selection errors, and docking errors. The order of presentation of the two input devices was balanced among participants, and each participant was presented with the same collection of target and dock size and locations. In summary, the design was:

\author{
12 participants $\mathrm{x}$ \\ 2 input devices (mouse, touch-table) $\mathrm{x}$ \\ 8 blocks of trials $\mathrm{x}$ \\ 16 repetitions \\ $=3072$ trials in total.
}

\section{Results}

None of our 12 participants had any previous experience with bimanual input using either two mice or two fingers on a touch-table. Since this was a new task for these individuals, one should expect to see strong learning effects as they become more comfortable with this type of input over the course of the experiment. Indeed, an ANOVA of the recorded data showed a strong learning effect for selection-time and error rates across blocks. Only after removing the first five blocks of trials did block lose its significant effect on these measurements. Therefore, the results in this section include only data collected in the final three blocks of the experiment.

\section{Selection-Time Analysis}

Selection-time was measured from the moment that the target and dock appeared on screen to the moment that both corner handles were successfully selected. Input-device had a significant main effect on selection-time $\left(\mathrm{F}_{1,11}=46.30, \mathrm{p}\right.$ $<0.001$ ), with mean selection times of $1.45 \mathrm{~s}$ and $2.43 \mathrm{~s}$ for touch-table and mouse input respectively.

\section{Selection-Error Analysis}

To select a target, the participant had to select two handles in opposite corners of the target with either their two fingers or two mouse pointers depending on the input condition. Missing one or both of the corner handles resulted in a 
selection-error. Participants committed significantly more errors when selecting targets with their fingers on the touch-table than when using two-mice $\left(\mathrm{F}_{1,11}=4.87, \mathrm{p}=\right.$ $0.05)$. The mean selection error rates were $18.9 \%$ and $9.7 \%$ for touch-table and mouse input respectively.

\section{Docking-Time Analysis}

The docking time was recorded as the time between the successful selection of the target and the successful docking of the target. The target docked automatically when each side of the target was within 5 pixels of the dock. Inputdevice had a significant main effect on docking-time $\left(\mathrm{F}_{1,11}\right.$ $=27.69, \mathrm{p}<0.001)$, with mean docking times of $2.10 \mathrm{~s}$ and $3.07 \mathrm{~s}$ for touch-table and mouse input respectively.

\section{Docking-Error Analysis}

Docking errors were rare and statistically indistinguishable between input devices $\left(\mathrm{F}_{1,11}=0.94, \mathrm{p}=0.35\right)$. The mean error rates were $0.9 \%$ and $1.9 \%$ for touch-table and two mice input respectively.

\section{Trial-Time Analysis}

Input-device had a significant main effect on trial time $\left(\mathrm{F}_{1,11}\right.$ $=31.90, \mathrm{p}<0.001)$ with mean trials times of $3.59 \mathrm{~s}$ and $5.56 \mathrm{~s}$ for touch-table and two mice input. The mean trial times for each input device are shown in Figure 13.



Figure 13. Mean bimanual trial times for each input device, broken down by selection time and docking time.

\section{Preference}

We again asked each participant which input condition they preferred for this task. Having used the system for around 45 minutes, they were in a good position to balance ease of use with fatigue in making their decision. A Wilcoxon signed ranks test indicated that subjects significantly preferred the touch-table for this bimanual task $(Z=-2.3, p$ $=0.02$ ), with ten of our twelve participants choosing the touch-table as their preferred input device.

\section{Experiment 2 Discussion}

Participants appeared to have trouble managing two indirect input devices, with several complaining that following two mouse pointers was very difficult to do. This difficulty seemed to lead to much higher selection-times for the twomouse input condition. In this experiment, both mice used the same control-display gain. One participant suggested that the left mouse be made less sensitive to movement since he had less practice using a mouse with his nondominant hand. This suggestion is interesting in that it points to differences between asymmetric and symmetric bimanual interaction [4, 23, 24]. Although our experiment task was a symmetric one, many bimanual interactions in the real world are asymmetric [14], with the non-dominant hand playing a less spatially precise and working at a lower temporal pace than the dominant hand. This participant's comment might be reflective of this prior real-world bimanual experience, and indicates that even though a virtual interaction might be symmetric and suggest that both input devices have similar characteristics, it could still be influenced by the asymmetric assumptions of the user.

Splitting one's focus between two independent graphical pointers during the target selection phase of a trial seemed to lead to relatively high selection times in the two-mouse condition. This reinforces the findings of Balakrishnan and Hinckley [4], who propose that the objects tracking the two mice be somehow visually connected to improve performance. During the docking phase of a trial, the target itself provided just such a visual connection; however, twomouse input resulted in significantly lower performance compared with two-finger input on the touch-table. As in Experiment 1, the consequence of a selection error was low with the only penalty being that the participant had to try selecting the target again. A more consequential result of a missed selection may have led to slower selection times as participants tried to avoid a costly error.

Target distance was not a controlled variable in Experiment 2 ; rather, targets were randomly distributed around the table. Because of the relationship between target distance and error rate found in Experiment 1, we performed an analysis of this relationship for Experiment 2 as well. We sorted all trials into five bins based on the distance between the target and fingers at the start of a trial. An ANOVA indicates that distance to target has a significant effect on selection error for touch-table input $\left(\mathrm{F}_{4,55}=5.32, \mathrm{p}=0.001\right)$ but has no significant effect on the error rate of two mouse input $\left(\mathrm{F}_{4,55}=0.53, \mathrm{p}=0.72\right)$. The mean error rates for each input device for the five ranges of target distances are shown in Figure 14. While two mouse input performed constantly across distances, the accuracy of two finger input decreased rapidly with distance. Of particular note is that, as in Experiment 1, our participants committed zero errors for targets located at the closest distance.

A task or input device that allows users to work their hands in parallel should outperform one that does not; thus, it is typical in bimanual evaluations to look at the amount of parallel hand movement as a measure of the efficiency of the bimanual task. We first looked at the mean time between the selection of the upper-right and lower-left handles of the target. These times were small for both touch-table $(0.26 \mathrm{~s})$ and two mice input $(0.21 \mathrm{~s})$. Participants seemed to position both pointing devices over the corners of the target before simultaneously selecting them. Because our touch-table did not sense tracking input, we were 
unable to compare movement toward the target during the selection phase of the trials; however, we were able to compare the amount of parallel input in the docking stage. We measured the distance between the second corner of the target when the first corned was aligned with the dock. In a perfectly executed trial, both corners of the target should align with the dock at the same moment and this distance would be zero. Touch-table input resulted in significantly more parallelism between corner placement that two mice input $\left(\mathrm{F}_{1,11}=30.46, \mathrm{p}<0.001\right)$. The mean distance between the trailing corner and the dock was 17.9 pixels for touchtable and 38.7 pixels for two mice input. This difference in parallelism is a strong candidate for explaining the differences in docking times: participants were more efficient at docking in this bimanual task when using their hands directly on the table.



Figure 14. Mean bimanual selection error rates for five ranges of target distances for both input devices.

\section{CONCLUSION}

Taken as a pair, Experiments $1 \& 2$ indicate that users may be better off using a mouse for unimanual input and their fingers for bimanual input when working on a large, horizontal display. It appears that system designers need to consider the proportion of unimanual and bimanual input that their system requires when choosing between directtouch and multi-mouse input. While a direct-touch input modality may not lead to greater performance in terms of speed and accuracy for unimanual tasks, other considerations, such as fatigue, spatial memory, and awareness of other's actions in a multi-user setting, might convince a system designer to choose single-finger input over single-mouse input in a tabletop environment. Further investigations into the qualities of tabletop devices are needed to quantify the costs and benefits of these characteristics.

\section{REFERENCES}

1. Accot, J. and Zhai, S. (1997). Beyond Fitts' law: models for trajectory-based HCI tasks. ACM CHI Conference on Human Factors in Computing Systems. p. 295-302.

2. Albinsson, P. and Zhai, S. (2003). High precision touch screen interaction. ACM CHI Conference on Human Factors in Computing Systems. p. 105-112.

3. Balakrishnan, R. and Hinckley, K. (1999). The role of kinesthetic reference frames in two-handed input performance. ACM UIST Symposium on User interface Software and Technology. p. 171-178.

4. Balakrishnan, R. and Hinckley, K. (2000). Symmetric bimanual interaction. ACM CHI Conference on Human Factors in Computing Systems. p. 33-40.

5. Barnert, W.C. (2005). A Comparison of One-Handed and Two-Handed Direct and Indirect Computer Interaction. Department of Computer Science, Tufts University, Medford, Mass. Technical Report 2005-11.

6. Benko, H., Wilson, A., and Baudisch, P. (2006). Precise selection techniques for multi-touch screens. $A C M C H I$ Conference on Human Factors in Computing Systems. p. 1263-1272.

7. Buxton, W. (1990). A three-state model of graphical input. IFIP TC13 Third Interantional Conference on Human-Computer Interaction. p. 449-456.

8. Buxton, W., and Myers, B. (1986). A study in twohanded input. ACM CHI Conference On Human Factors in Computing Systems. p. 321-326.

9. Card, S., English, W., and Burr, B. (1978). Evaluation of mouse, rate-controlled isometric joystick, step keys, and text keys for text selection on a CRT. Ergonomics, 21. p. 601-613.

10. Czerwinski, M., Smith, G., Regan, T., Meyers, B., Robertson, G., and Starkweather, G. (2003). Toward characterizing the productivity benefits of very large displays. Interact Conference. p. 9-16.

11. Esenther, A. and Ryall, K. (2006). Fluid DTMouse: Better mouse support for touch based interactions. Advanced Visual Interfaces Conference. p. 112-115.

12. Fitts, P. (1954). The information capacity of the human motor system in controlling the amplitude of movement. Journal of Experimental Psychology, 47. p. 381-391.

13. Guiard, Y. (2001). Disentangling relative from absolute amplitude in Fitts' law experiments. $A C M C H I$ Conference on Human Factors in Computing Systems, Extended Abstracts. p. 315-316.

14. Guiard, Y. (1987). Asymmetric division of labor in human skilled bimanual action: the kinematic chain as a model. Journal of Motor Behavior, 19(4). p. 486-517.

15. Guiard, Y., and Ferrand, T. (1995). Asymmetry in bimanual skills. in Manual asymmetries in motor performance, Elliott and Roy, Editors. CRC Press.

16. Grossman, T., and Balakrishnan, R. (2005). A probabilistic approach to modeling two-dimensional pointing. ACM Transactions on Computer-Human Interaction, 12(3). p. 435-459.

17. Hinckley, K., Czerwinski, M., and Sinclair, M. (1998). Interaction and modeling techniques for desktop twohanded input. ACM UIST Symposium on User Interface Software and Technology. p. 49-58. 
18. Hinckley, K., Pausch, R., Proffitt, D., and Kassell, N. (1998). Two-handed virtual manipulation. ACM Transactions on Computer-Human Interaction, 5(3). p. 260-302.

19. Hinckley, K., Pausch, R., Proffitt, D., Patten, J., and Kassell, N. (1997). Cooperative bimanual action. ACM CHI Conference on Human Factors in Computing Systems. p. 27-34.

20. Kabbash, P., Buxton, W., and Sellen, A. (1994). Twohanded input in a compound task. ACM CHI Conference on Human Factors in Computing Systems. p. 417-423.

21. Kelso, J., Southard, D., and Goodman, D. (1979). On the coordination of two-handed movements. Journal of Experimental Psychology: Human Perception and Performance, 5(2). p. 229-238.

22. Kurtenbach, G., Fitzmaurice, G., Baudel, T., and Buxton, B. (1997). The design of a GUI paradigm based on tablets, two-hands, and transparency. ACM CHI Conference on Human Factors in Computing Systems. p. $35-42$.

23. Latulipe, C., Kaplan, C., and Clarke, C. (2005). Bimanual and unimanual image alignment: an evaluation of mousebased techniques. ACM UIST Symposium on User interface Software and Technology. p. 123-131.

24. Latulipe, C., Mann, S., Kaplan, C., and Clarke, C. (2006). SymSpline: symmetric two-handed spline manipulation. ACM CHI Conference on Human Factors in Computing Systems. p. 349-358.

25. Leganchuk, A., Zhai, S., and Buxton, W. (1999). Manual and cognitive benefits of two-handed input: an experimental study, ACM Transactions on ComputerHuman Interaction, 5(4). p. 326-359.

26. MacKenzie, S. (1992). Fitts' law as a research and design tool in human-computer interaction. Human-Computer Interaction, 7. p. 91-139.

27. MacKenzie, S. and Buxton, W. (1992). Extending Fitts' law to two-dimensional tasks. ACM CHI Conference on Human Factors in Computing Systems. p. 219-226.

28. MacKenzie, S., Sellen, A., and Buxton, W. (1991). A comparison of input devices in elemental pointing and dragging tasks. ACM CHI Conference on Human Factors in Computing Systems. p. 161-166.

29. MacKenzie, S., and Soukoreff, W. (2003). Card, English, and Burr (1978) -- 25 years later. ACM CHI Conference on Human Factors in Computing Systems, Extended Abstracts. p. 760-761.

30. Marteniuk, R., MacKenzie, C., and Baba, D. (1984). Bimanual movement control: Information Processing and interaction effects. Quarterly Journal of Experimental Psychology, 36A. p. 335-365.
31. Meyer, S., Cohen, O. and Nilsen, E. (1994). Device comparisons for goal-directed drawing tasks. ACM CHI Conference on Human Factors in Computing Systems, Extended Abstracts. p. 251-252.

32. Microsoft. Pointer ballistics for Windows XP. Accessed on Mar 23, 2006, http:/www.microsoft.com/ whdc/device/input/pointer-bal.mspx.

33. Mine, M., Brooks, F., and Sequin, C. (1997). Moving objects in space: expoiting proprioception in virtualenvironment interaction. ACM SIGGRAPH Conference on Computer Graphics and Interactive Techniques. p. 1926.

34. Morris, M. (2006). Supporting effective interaction with tabletop groupware. Ph.D. Dissertation, Department of Computer Science, Stanford University.

35. Owen, R., Kurtenbach, G., Fitzmaurice, G., Baudel, T., and Buxton, W. (2005). When it gets more difficult, use both hands: exploring bimanual curve manipulation. Graphics Interface Conference. p. 17-24.

36. Parker, K., Mandryk, R., Nunes, M., and Inkpen, K. (2005). TractorBeam selection aids: Improving target acquisition for pointing input on tabletop displays. Interact Conference. p. 80-93.

37. Scott, S. (2005). Territoriality in collaborative tabletop workspaces. Ph.D. Dissertation, Department of Computer Science, University of Calgary.

38. Scott, S. and Carpendale, S. (2006). Guest editors' introduction: interacting with digital tabletops. IEEE Computer Graphics \& Applications: Special Issue on Interacting with Digital Tabletops, 26(5). p. 24-27.

39. Sears, A. and Shneiderman, B. (1991). High precision touchscreens: design strategies and comparisons with a mouse. International Journal of Man-Machine Studies, 34(4). p. 593-613.

40. Shneiderman, B. (1991). Touchscreens now offer compelling uses. IEEE Software, 8(2). p. 93-94.

41. Wu, M. and Balakrishnan, R. (2003). Multi-finger and whole hand gestural interaction techniques for multi-user tabletop displays. ACM UIST Symposium on User interface Software and Technology. p. 193-202.

42.Zeleznik, R., Forsberg, A., and Strauss, P. (1997). Two pointer input for 3D interaction. ACM Symposium on Interactive $3 D$ Graphics. p. 115-120.

43. Zhai, S. (2004). Characterizing computer input with Fitts' law parameters - The information and non-information aspects of pointing. International Journal of HumanComputer Studies, 61(6). p. 791-809. 